\title{
Investigation of finite beta modified drift wave in a tokamak plasma
}

\author{
E. D. Fredrickson a) and P. M. Bellan \\ California Institute of Technology, Pasadena, California 91125
}

(Received 20 August 1984; accepted 3 February 1985)

\begin{abstract}
The saturated state of a low-frequency, coherent, $m=2$, global mode has been studied in the Encore tokamak using probe techniques. The mode is found to have large fluctuations in density, electron temperature, space potential, and magnetic field. The experimental measurements were compared with the predictions of a computer code, based on a linear, two-fluid theory of the coupling of drift and shear-Alfvén modes. This comparison led to the identification of the mode as a finite beta modified drift wave.
\end{abstract}

\section{INTRODUCTION}

One of the likely causes of the anomalous transport in tokamaks is the magnetic and density fluctuations. "These fluctuations can be classified into two general types: lowfrequency, long wavelength coherent modes (often called Mirnov oscillations) and higher frequency, shorter wavelength, strongly turbulent fluctuations. The Mirnov oscillations are generally considered to be primarily magnetic peturbations and the derivations generally assume the plasma is incompressible. ${ }^{2}$ The turbulence is in the drift range of frequencies, and nonlinear saturated states of drift or driftAlfvén waves are considered to be strong candidates for an explanation of these fluctuations.

Strong coherent fluctuations in density and magnetic field have been observed experimentally on the Encore tokamak. Probe measurements show that this mode nas large fluctuations in density, electron temperature, space potential, and magnetic field. The poloidal and toroidal mode numbers, as well as the radial structure of the fluctuations, have been measured. The equilibrium plasma characteristics were also determined with probe measurements. Magnetic probes were used to determine the radial profile of the poloidal magnetic field, from which the rotational transform and current density profiles could be deduced. A Langmuir probe was used to measure radial profiles of the density, electron temperature, and space potential.

A numerical code was developed to solve a linearized set of the two-fluid equations, simplified by the assumption of low frequency, $\omega<\omega_{c i}$, and other assumptions consistent with the experimental parameters. The code solves the resulting reduced set of equations in bounded cylindrical geometry. A similar set of equations has been used by previous authors to investigate the short wavelength drift-Alfvén modes. ${ }^{3-6}$

In a warm magnetized plasma these equations have two types of solutions, the ion-acoustic waves and the shear-Alfvén waves. In the presence of a density gradient perpendicular to the magnetic field, the ion-acoustic wave is modified to become the drift wave, which is basically electrostatic and propagates nearly perpendicular to the magnetic field. ${ }^{7}$ The shear-Alfvén waves are electromagnetic waves in which the

- Present address: Princeton Plasma Physics Laboratory, Princeton University, Princeton, New Jersey 08544. ion mass provides the inertial force and the resistance of the magnetic field lines to bending provides the restoring force. ${ }^{8}$ They propagate nearly parallel to the magnetic field at approximately the Alfven velocity, $v_{\mathrm{A}} \equiv B\left(4 \pi n m_{i}\right)^{-1 / 2}$. When the parallel phase velocity of the drift wave approaches $V_{A}$, the drift and Alfvén branches become coupled and the drift wave acquires a strong magnetic component.

Of the two shear-Alfvén solutions and the drift branch solution, it was found that the drift wave solution best fit the observed frequency of the mode and the relative amplitudes of the density, space potential, and magnetic fluctuations. The shear-Alfven solutions had much larger magnetic fluctuations for a given level of density fluctuations. The identification of the experimentally observed $m=2$ mode as a finite $\beta_{T}\left(\equiv 8 \pi n T / B^{2}\right)$ modified drift wave means that the mode is more closely related to the higher frequency, turbulent fluctuations observed on larger machines, rather than the lower frequency coherent Mirnov oscillations.

Previously, similar modes with large, well-correlated density and magnetic fluctuations have been observed on linear arcjet plasma devices at Nagoya ${ }^{9}$ and at UCLA. ${ }^{10}$ These machines also operate at parameters similar to those in Encore. They are physically smaller, and observations on the modes were limited to comparison of the observed scaling of frequencies and amplitudes of the fluctuating quantities to the predictions of a local theory.

In Sec. II the machine and diagnostics will be described and the results of the experimental measurements presented. In Sec. III the linear equations describing the coupling of the drift and Alfvén branches will be derived and a simplified version will be solved analytically. Numerical solution of the equations will be discussed and the results of the calculations will be presented. In Sec. IV the results will be discussed and some conclusions presented.

\section{EXPERIMENT}

\section{A. Description of the Encore tokamak}

The experiments were performed on the California Institute of Technology Encore tokamak. The principal features of this machine are the high pulse repetition rate, typically 15 discharges per second, and the fact that the plasma is cold enough that probes can be inserted as far as the center of the discharge. The high repetition rate is achieved by using a steady-state toroidal field (variable from 0.1 to $1.5 \mathrm{kG}$ ) and a high power audio-frequency amplifier as the Ohmic heating 
power supply. The maximum plasma current is limited to about $10 \mathrm{kA}$ by the power available from the $\mathrm{OH}$ amplifier, but in practice, the requirement that the probes survive the heat load from the plasma limits the plasma current to less than about $4 \mathrm{kA}$. Typical densities and electron temperatures were $n \approx 1-2 \times 10^{12} \mathrm{~cm}^{-3}$ and $T \approx 10-15 \mathrm{eV}$. The length of the discharges was $\approx 4 \mathrm{msec}$, variable from 3-6 msec.

The $1.27 \mathrm{~cm}$ thick aluminum vacuum chamber walls provide some stabilization for the plasma; a pulsed vertical field makes up for flux lost in the resistive decay of the wall currents. Access to the plasma is provided by $33 \mathrm{small}(4 \mathrm{~cm}$ diam) and 15 large $(8 \times 22 \mathrm{~cm})$ ports. The plasma minor radius is $12.6 \mathrm{~cm}$, with the wall acting as a limiter. The major radius is $38.1 \mathrm{~cm}$, resulting in an aspect ratio of three.

The plasma energy loss is dominated by line radiation from the neutral or partially stripped gas. Estimates of the radiated power based on the calculations of Post et al. ${ }^{11}$ show that radiation can account for nearly all the input Ohmic heating power. In the highest current hydrogen discharges the plasma does burn through this radiation dominated regime, but the discharges were not sufficiently reproducible for making discharge-to-discharge measurements, and were also hot enough to destroy any probes exposed for short periods of time to the plasma. For these reasons it was not practical to do experiments in burn-through discharges. Argon, being easier to ionize, gave more reproducible discharges and was typically used as the filling gas. Argon plasmas also did not have the high levels of $x$-ray radiation encountered in hydrogen discharges. Discharges run with hydrogen, helium, and nitrogen were similar to the argon discharges, but were less reproducible.

The practical limits on plasma current, and hence density and temperature, were set by the requirements that the plasma break down reproducibly and that the probes could withstand the heat flux resulting from the high duty cycle $(\sim 8 \%)$. The acceptable range of plasma currents was then about 1.5 to $4.0 \mathrm{kA}$, the lower limit was set by the requirement of consistent breakdown, and the upper limit by the maximum tolerable heat flux to the probes. For the data

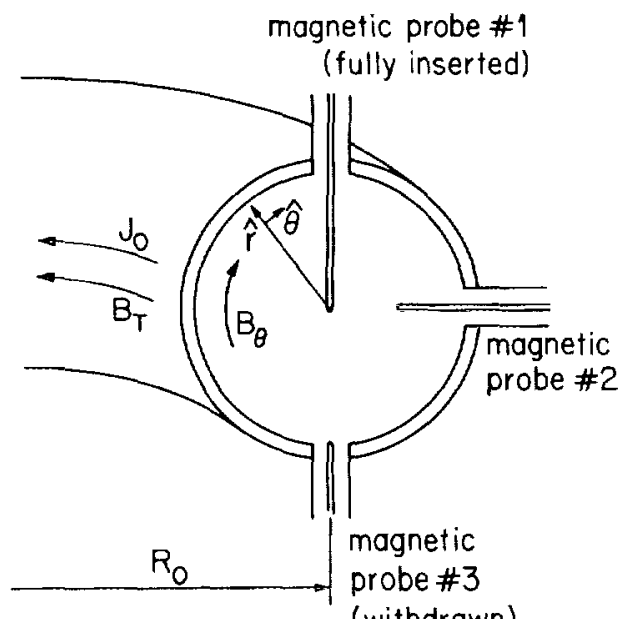

FIG. 1. This figure shows the orientation of the three magnetic probes described in Sec. II. Also shown are the directions of the toroidal field, plasma current, and poloidal field.

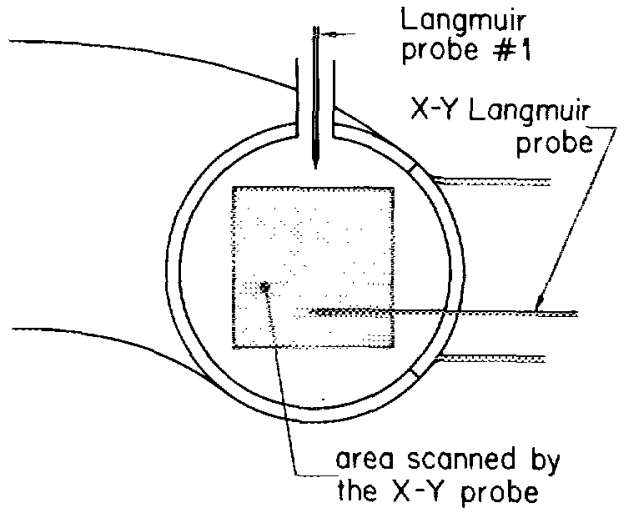

FIG. 2. This figure shows the orientation of the two Langmuir probes described in Sec. II. The shaded region shows the region of travel of the $X-Y$ probe.

presented in this work, a $q$ at the wall of between three and four was desirable, where $q(r)$ for a cylindrical tokamak is defined as ${ }^{12}$

$$
q(r)=\frac{\partial \Psi_{\text {tor }}}{\partial \Psi_{\mathrm{pol}}} \approx \frac{r B_{T}}{R B_{\theta}(r)}
$$

Here $r$ is the minor radius, $R$ is the major radius, $B_{T}$ is the toroidal field, and $B_{\theta}$ is the poloidal field (as shown in Fig. 1). Thus the typical toroidal field strengths were between 250 and $400 \mathrm{G}$. For these parameters, $\beta_{T} \equiv 8 \pi n T / B^{2} \approx 0.01-$ 0.02 . Within this range of parameters, it was possible to obtain reproducible discharges relatively insensitive to the presence of probes.

The principal plasma diagnostics were two moveable Langmuir probes and three moveable magnetic probes (see Figs. 1 and 2). The magnetic probes consisted of small, air cooled coils, oriented so that the time integrated signal gave the local poloidal magnetic field strength. The probes were mounted on stepper motor driven probe drives, controlled by an LSI-11/23 computer. One probe drive (the $X-Y$ drive) could scan a probe over most of the plasma minor cross section (see Fig. 2).The other probe drives (the linear drives) moved the probes only in the radial direction. The magnetic probes and one Langmuir probe were mounted on the linear probe drives and a second Langmuir probe was mounted on the $X-Y$ probe drive.

\section{B. Measurement of the equilibrium profiles}

Oscilloscope traces of the plasma current, loop voltage, and probe signals are presented in Fig. 3 and a typical set of data showing the equilibrium safety factor, density, electron temperature, and space potential profiles is presented in Fig. 4. The data presented in these figures were collected over a period of about 30 minutes of machine running time, corresponding to $\approx 30000$ discharges. Data points were collected in only a small fraction of these discharges, $<5000$; most of the time was spent in moving the probes and in transferring, processing, and making permanent records of the data. The plasma was highly reproducible; variations in global quantities such as plasma current, loop voltage, and density were typically less than a few percent over the 30000 discharges. Likewise, the profiles of density, poloidal field, electron temperature, etc., were also reproducible. 

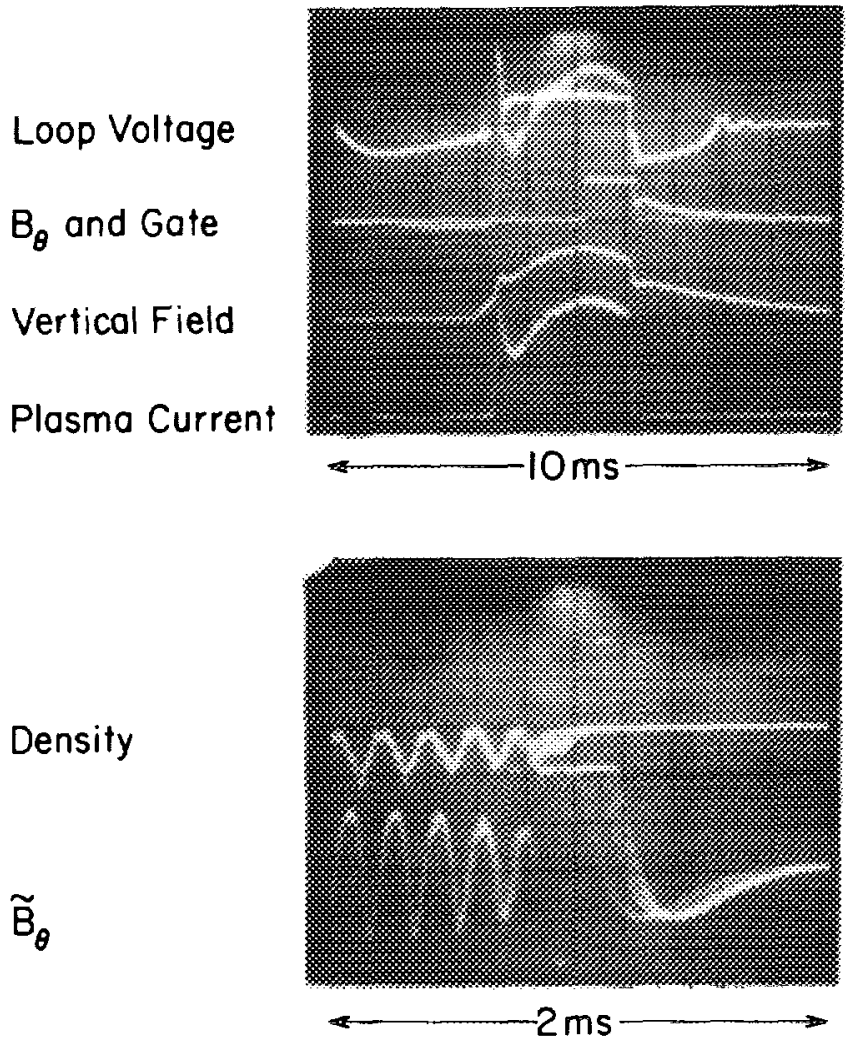

FIG. 3. The upper oscilloscope photograph shows the typical loop voltage (10 V/div), poloidal magnetic field at the wall (as measured with a magnetic probe), vertical field current ( $16 \mathrm{~A} / \mathrm{div})$, and plasma current $(2 \mathrm{kA} /$ div $)$. The time axis is $1 \mathrm{msec} / \mathrm{div}$ or $10 \mathrm{msec}$ full scale. The lower photograph shows the density and magnetic fluctuations on an expanded scale, $0.2 \mathrm{msec} / \mathrm{div}$ or 2 msec full scale.

The density, space potential, and electron temperature (solid curve) were measured with the Langmuir probe. The electron temperature profile with the dashed line was computed from the plasma current density profile, assuming Spitzer resistivity ${ }^{13.14}$ and using $Z_{\text {eff }}=4.5\left(Z_{\text {eff }}\right.$ is the average charge state of the ions), i.e.,

$$
T_{e}=5.3\left[Z_{\text {eff }} J(r) / V_{\text {loop }}\right]^{2 / 3},
$$

where $T_{e}$ is in $\mathrm{eV}$ and $J(r)$ is in $\mathrm{A} / \mathrm{cm}^{2} ; J(r)$ is computed from the measured $B_{\theta}$ profile. The choice of $Z_{\text {eff }}=3-6$ is based on a comparison of the plasma conductivity and the Langmuir probe measurement of the electron temperature and is found to be consistent with the predictions of Post et al. ${ }^{11}$ The two measurements are in rough agreement, but the shape of the profiles is different. The error in the conductivity measurement was less than $10 \%$, and the error in the Langmuir probe temperature was less than $5 \%$, so these measurements would indicate that the $Z_{\text {eff }}$ of the plasma was a function of the minor radius.

The density, temperature, and space potential were determined from measurements of the ion saturation current and a sixteen point $I-V$ characteristic for Langmuir probe 1 in Fig. 2. The sixteen bias voltages for the $I-V$ characteristic were chosen so that the temperature of the bulk electrons (i.e., not the tail of the distribution function), would be measured. The temperature was calculated from the data by a least squares fit to the theoretical Langmuir probe character-
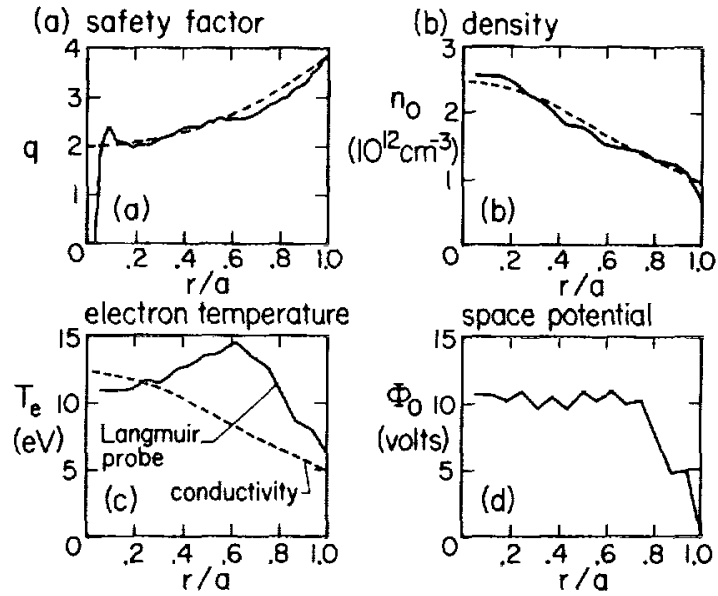

FIG. 4. Radial profiles of the (a) safety factor, $q(r)$, (b) density, (c) electron temperature, and (d) space potential. The broken lines in Figs. $4(\mathrm{a}), 4(\mathrm{~b})$, and 4 (c) are the profiles used in the numerical code when computing the results presented in Fig. 11. The tocoidal field for these data was $350 \mathrm{G}$.

istic. This fit also predicted the plasma space potential. The presence of the magnetic field was assumed not to affect the Langmuir probe characteristic over the range of the bias voltages chosen. ${ }^{15}$ The density was then determined by measuring the ion saturation current and using

$$
I_{\text {sat }}=n e c_{s} A \text {, }
$$

where $n$ is the local electron density, $c_{s}=\left(T_{e} / m_{i}\right)^{1 / 2}$, and $A$ is the area of the probe. The Langmuir probe measurements of density were compared to the line averaged density of the plasma measured by a $3 \mathrm{~mm}$ microwave interferometer and were found to be in good agreement.

As measurement of the absolute space potential in a magnetized plasma with a Langmuir probe is difficult, the common practice is to relate fluctuations in the floating potential with space potential fluctuations. The presence of strong fluctuations in the electron temperature meant that this practice was not valid, and thus necessitated this direct measurement of the space potential.

The magnetic probes were used to measure the timedependent poloidal magnetic field. Because the amplitude of the magnetic fluctuations was $\leqslant 5 \%$ of the dc poloidal field, the fluctuating field component was amplified and digitized independently of the total poloidal field. The total poloidal field was then averaged over time to give the dc poloidal field component. This time-averaged magnetic field data could be used to calculate an approximate safety factor profile $q(r)$. When $q(r)$ is equal to a rational number $m / n$, the magnetic field structure will be resonant with a mode having poloidal mode number $m$ and toroidal mode number $n$ [i.e., $\mathbf{k} \cdot \mathbf{B}=0$, where $\mathbf{k} \equiv(m / r) \hat{\theta}+(n / R) \hat{\varphi}$.] The stability and structure of low- $m$ modes is strongly affected by the presence of these mode rational surfaces.

In principle, it is necessary to know the complete toroidal and poloidal structure of the magnetic fields to calculate $q(r)$. In practice, it is a good approximation to neglect any toroidal dependence in the time-averaged fields, and then make some assumption about the poloidal dependence of the poloidal magnetic field. The simplest assumption is that the flux surfaces are circular and concentric, which is equivalent 
to the assumptions that plasma $\beta_{T}<1$ and the aspect ratio $R / a$ is large ${ }^{16}(R$ and $a$ are, respectively, the major and minor radii of the vacuum vessel). With these assumptions, the expression for the safety factor in terms of the poloidal magnetic field measured from the top or bottom (probes 1 and 3 in Fig. 1) of the machine is

$$
q(r)=\left[r B_{T} / R B_{\theta}(r)\right]\left[1-(r / R)^{2}\right]^{-1 / 2} .
$$

For measurements made at other poloidal angles, the expression for $q(r)$ is slightly different, but in general, near $r=0$, $q(r)$ is very nearly the same as for the straight cylinder case.

\section{Measurement of the mode structure}

Strong, coherent fluctuations in density and magnetic field were observed over a wide range of plasma parameters. The discharges in which these waves were seen can be divided into three categories: those with $q(0) \approx 1$, and with $q(0) \approx 2$, and those with $q(0)>2$. This work will deal primarily with discharges in the second category, $q(0) \approx 2$. In the discharges with $q(0) \approx 1$, it was observed that the density fluctuations had a dominant poloidal mode number of one, but that the magnetic fluctuations were dominantly $m=2$. The coupling of different poloidal modes by toroidal geometry is of considerable interest, but the equations involved are more complex than those for a single poloidal mode. For this reason, although many experimental measurements were made, no theoretical calculations will be discussed describing these discharges. In discharges with $q(0)>2$, the experimental measurements were harder to interpret as there was strong evidence that more than one poloidal mode was present and the modes tended to be localized more to the periphery of the plasma (where it was difficult to make good measurements). Thus, the discharges with $q(0) \approx 2$ offered the greatest possibility for successful comparison of theory to experiment.

A typical data set showing the mode structure for discharges with $q(0) \approx 2$ is presented in Figs. 5-9. In these discharges a coherent mode (or wave) was observed with a frequency of $\sim 2-5 \mathrm{kHz}$. These modes had a poloidal mode number of $m=2$ and a toroidal mode number of $n=1$. The

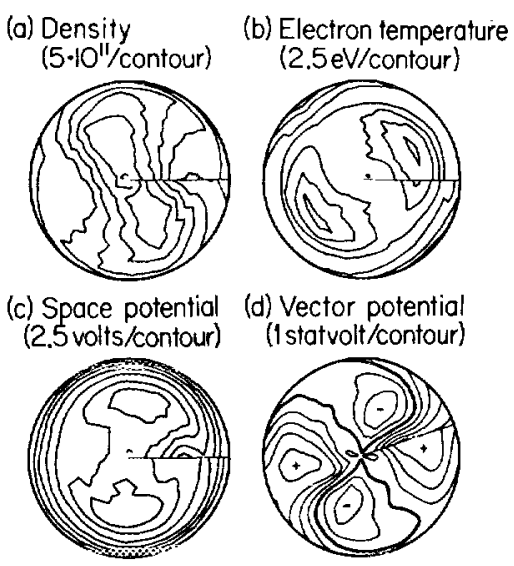

FIG. 5. Contour plots of the (a) density, (b) electron temperature, (c) space potential, and (d) vector potential. The figures are plotted such that the poloidal angle corresponds to the time axis, scaled so that $2 \pi$ rads corresponds to twice the wave period. The contour plots then approximate the actual spatial structure of the modes. The solid line corresponds to $t=0$.
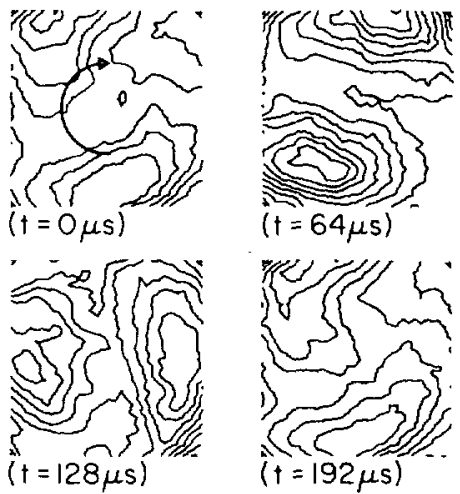

FIG. 6. Contour plots of the floating potential as measured with the $X-Y$ probe. Each plot corresponds to a different time delay with respect to a fixed reference. The electron diamagnetic drift direction is shown by the arrow; thus the mode is seen to rotate in the electron diamagnetic drift direction.

$q$ at the center of the plasma was approximately 2 . Within the errors in calibration it was not possible to determine whether or not a mode rational surface actually existed in the plasma; if it did it was very near the plasma center. Thus the $m=2$ mode was not centered on a rational surface as would be expected for a tearing type of instability.

The time dependence of the fluctuating quantities was measured by digitizing the signal over a time period somewhat longer than the period of the mode oscillations. The Langmuir probe measurements were digitized over a period of typically $512 \mu \mathrm{sec}$, with 16 samples at $32 \mu \mathrm{sec}$ intervals. The magnetic fluctuations were digitized with a different instrument: the data collection interval was $640 \mu \mathrm{sec}$, with 32 samples at $20 \mu \mathrm{sec}$ intervals. Each data point was additionally averaged over four discharges, and the spatial structure of the mode was determined by moving the probe to different spatial locations between. The Langmuir probe characteristic required measurements for sixteen different probe bias voltages as well as measurement of the ion saturation current and the floating potential. A set of data was then necessarily collected over many plasma discharges. To maintain a constant phase reference, a separate Langmuir probe signal was used as a reference to trigger the data collection system.
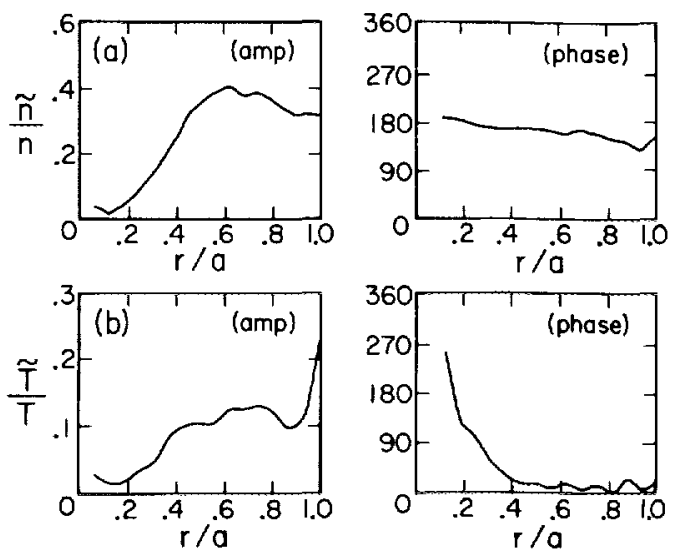

FIG. 7. Radial profiles of the amplitude and phase of the fundamental harmonic of the (a) density and (b) temperature fluctuations. The density and temperature fluctuations are normalized to the local average of the density and temperature respectively. 

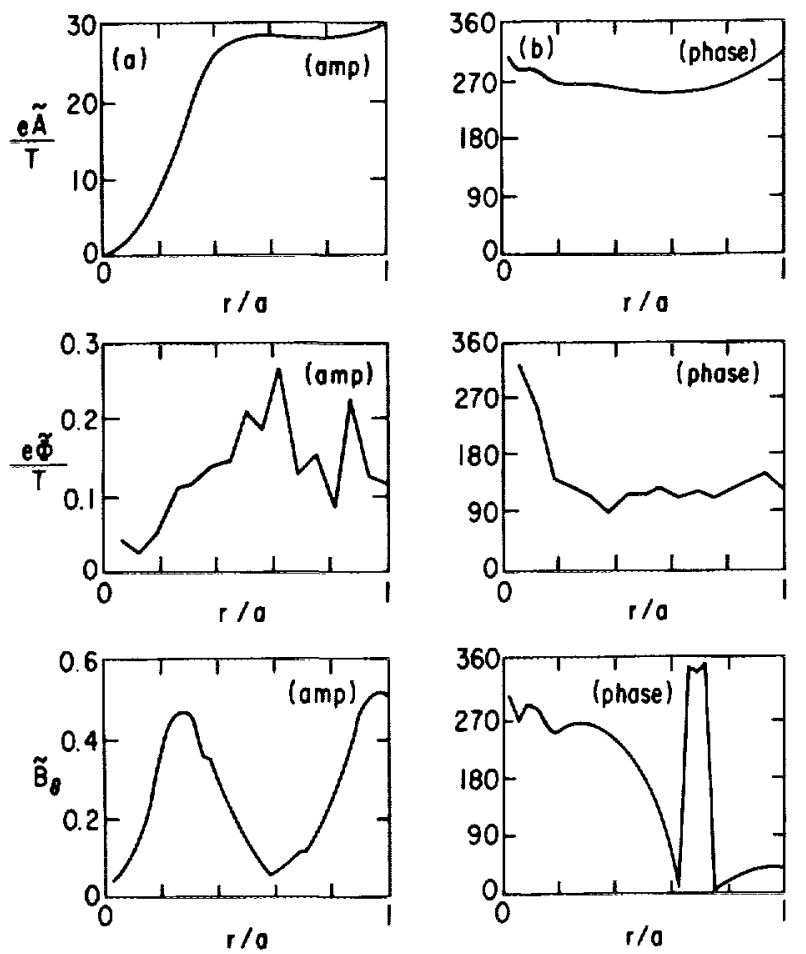

FIG. 8. Radial profiles of the amplitude and phase of the fundamental harmonic of the (a) parallel vector potential, and (b) space potential fluctuations, and (c) poloidal magnetic field fluctuations (in gauss). The vector potential and space potential fluctuations have been normalized to the local average electron temperature.

Contour plots of the density, temperature, space potential, and parallel component of the vector potential are presented in Fig. 5. As the mode is rotating, time can be mapped into poloidal angle: thus what appears as the poloidal angle in the plots is the time history of the mode measured at the fixed poloidal angle of the probe. As the parameters of the discharge and the amplitude of the mode are changing, the contours do not exactly match at $\theta=0$ and $2 \pi$.

The magnetic structure of the mode was investigated with three moveable magnetic probes located on the top, outside, and bottom of the machine. Each probe was moved along a minor radius from the center of the vacuum chamber to the wall. The time-dependent behavior of the magnetic
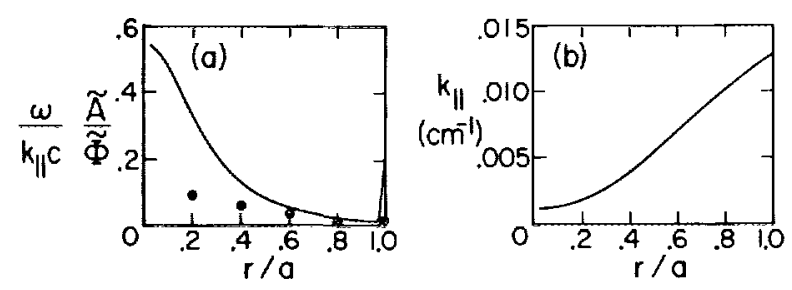

FIG. 9. (a) The ratio of the inductive component of the parallel electric field to the electrostatic component. The ratio varies from $\approx 0.1$ at the center of the plasma to $\approx 0.03$ at $r / a=0.8$. This ratio is a measure of the strength of the coupling of the drift and Alfvén branches. The solid curve is the theoretical prediction and the solid circles are from experimental measurements. The relative error in the calculations and the measurements becomes very large near $r \approx 0$ as $k_{\sharp}$ is not well known and the mode amplitudes are small; thus the discrepancy between the two sets of data is not significant in this region. Here (b) shows the variation of the parallel wave vector with the minor radius. field was measured at 32 radial positions. From the relative phase shifts in the fluctuating components of the magnetic field between the three probes it was possible to determine the dominant poloidal mode number of the magnetic fluctuations to within an additive factor of 4 . (The factor of 4 results from the minimum poloidal spacing of $90^{\circ}$ for the probes.) For the discharges with $q(0) \approx 2$, the dominant poloidal mode structure was $m=2$. The fluctuating magnetic field was spatially integrated from the center of the plasma to find the radial structure of the vector potential using the relation

$$
\widetilde{B}_{\theta}=(\nabla \times \mathbf{A})_{\theta} \approx-\frac{\partial \widetilde{A}_{\|}}{\partial r} .
$$

In this expression the components of the vector potential perpendicular to the magnetic field have been ignored (thus ignoring coupling to the compressional Alfvén waves). This approximation is valid in the limit of low $\beta_{T}$ discharges ${ }^{17}$ (here $\beta_{T} \approx 0.01-0.02<1$ ).

To investigate the electrostatic structure of the mode, two Langmuir probes were used. The first of these scanned a large part of the plasma minor cross section to determine the dominant poloidal mode number of the electrostatic fluctuations. Measurements were made of both the ion saturation current fluctuations and the floating potential fluctuations. The dominant poloidal mode structure of these was the same, that is, $m=2$ for the discharges with $q(0) \approx 2$. Since measurement of the floating potential was less perturbing to the plasma and less damaging to the probe, most of the subsequent measurements were made of the floating potential. By making measurements of the floating potential contours at several closely spaced times during the discharge, it was possible to determine the direction of the mode propagation. In Fig. 6 a set of this data is presented in which the rotation can be seen to be in the electron diamagnetic drift direction (arrow).

The radial profiles of the amplitudes of the density, electron temperature, vector potential, and space potential fluctuations, as well as the phase relative to a fixed reference, are presented in Figs. 7 and 8 . The ratio of the inductive to electrostatic components of the parallel electric field is presented in Fig. 9; for the experimental data (the filled circles in the figure) the maximum value of the ratio is $\approx 0.1$. The level of density fluctuations was very large, $\tilde{n} / n \approx 0.4$ at $r / a=0.6$. The amplitude of the vector potential fluctuations was found to be approximately 1.5 statvolts, corresponding to a fluctuation in $B_{\theta}$ of $0.5-1.0 \mathrm{G}$. The density fluctuations led the magnetic fluctuations by approximately $90^{\circ}$. The temperature fluctuations were out of phase by $180^{\circ}$ with the density fluctuations. This would be expected for a radiation-dominated discharge as the radiated power scales as $n^{2}$. The temperature fluctuation level was also large, with $\widetilde{T} / T \approx 0.13$ at $r / a=0.6$. This temperature fluctuation level is large enough to affect the local plasma resistivity, and therefore would be important in a theory describing the coupling of electrostatic to magnetic fluctuations. The space potential fluctuations were found to lead the density fluctuations by $\approx 45^{\circ}$; this phase shift corresponds to a large inward transport of the plasma and in the simple theory would normally be associated with damping of the mode. ${ }^{18}$ The amplitude of the space 
potential fluctuations is approximately $2.5 \mathrm{~V}$ at $r / a=0.6$, corresponding to $e \widetilde{\Phi} / T_{e} \approx 0.18$.

The measurements presented above are typical for all the $q(0) \approx 2$ discharges studied. The absolute level of the fluctuations was observed to vary somewhat, and in some cases the relative phases were not as cleanly defined as in this set of data. The toroidal field was varied over a range of 200 to 400 $\mathrm{G}$ in an attempt to determine the scaling of the frequency or mode amplitudes on $B_{T}$. The results were inconclusive; the frequency was found to vary, but not in a consistent fashion. Later numerical work also showed that the frequency was a weak function of toroidal field in this parameter range, but could depend strongly on other, less controllable plasma parameters. Likewise, no consistent scaling with $B_{T}$ of the relative mode amplitudes was observed.

\section{THEORY}

\section{A. Introduction}

In this section a theoretical model will be derived and its predictions will be compared to the experimental measurements discussed in the previous section. This model is an extension of those used previously by other authors ${ }^{3-6}$ to describe high- $m$ drift and drift-Alfvén modes. These high- $m$ modes are found to be localized to regions near the rational surface; the geometry may then be simplified to that of an infinite sheared slab. In the present work it is necessary to include the full bounded sheared cylindrical geometry because the radial wavelength is of order of the machine minor radius. The equations will also include the current gradient terms, which are important for determining the stability of low- $m$ tearing modes, and will include temperature fluctuations in a manner similar to that of Ellis and Motley. ${ }^{19}$

The model is valid for the description of modes with frequencies much below the ion-cyclotron frequency, $\omega_{c i}$, and for plasma parameters consistent with those found in tokamak edge plasmas or in small tokamaks such as Encore. The reduced set of equations which form the basis for the model are a set of three coupled differential equations relating the space potential $\widetilde{\Phi}$, the parallel component of the vector potential $\tilde{A}$, and the fluctuations in density $\tilde{n}$.

\section{B. Derivation of the reduced equations}

Moderate plasma $\beta_{T}$ is assumed, i.e., $m_{e} / m_{i}<\beta_{T}<1$. Thus the coupling of the drift and Alfvén branches is important, but the perpendicular components of the inductive electric field may be ignored, ${ }^{16}$ i.e., $\quad \mathbf{E}_{\|}=-\nabla_{\|} \tilde{\Phi}$ $-(1 / c)(\partial / \partial t) \tilde{\mathbf{A}}_{\|}$, but $\mathbf{E}_{1}=-\nabla_{1} \widetilde{\Phi}$. The mode frequency is assumed to lie in the range $v_{i e}<\omega<\omega_{c i}, v_{e i}$. Fluctuations in electron temperature, $\widetilde{T}$, which were observed in the Encore experiments, are included in an ad hoc fashion. The ions are assumed cold and the toroidal plasma will be approximated by a straight cylinder; the toroidal direction corresponds to $\hat{z}$ and the poloidal direction to $\hat{\theta}$. The perturbations are assumed to have the following form:

$$
F(r, \theta, z)=f(r) \exp ^{\left.\left[i \mid m \theta-k_{z^{z}}-\omega t\right)\right]} .
$$

Because of the low- $\beta_{T}$ assumption, fluctuations in the parallel magnetic field can be neglected, ${ }^{16}$ thus $\widetilde{\mathbf{B}}=\widetilde{\mathbf{B}}_{1}=\nabla \times \widetilde{A} \hat{b}$, so that only $\widetilde{\mathbf{A}}_{\|}$is necessary. Here parallel will be taken to mean parallel to the equilibrium magnetic field. The collision frequency is assumed to have the following dependence on density and temperature:

$$
v_{e i} \propto n T^{-3 / 2} \text {. }
$$

With the above assumptions, the linearized versions of the Braginski two-fluid equations are then ${ }^{20}$

$$
\begin{aligned}
0= & -n e\left(-\nabla \widetilde{\Phi}+i \frac{\omega}{c} \widetilde{A} \hat{b}\right)-\frac{e}{c} \widetilde{\Gamma}_{e} \times \mathbf{B}_{0}+\frac{1}{c} \mathbf{J}_{0} \times \widetilde{\mathbf{B}} \\
& -\nabla \widetilde{P}-\sigma n \nabla_{\|} \widetilde{T}-\frac{c m_{e} v_{e i}}{4 \pi e} \nabla_{1}^{2} \widetilde{A} \hat{b} \\
& -\frac{3 m_{e} v_{e i}}{2 e} J_{\| 0}(r)\left(\frac{\widetilde{T}}{T}\right) \hat{b} \\
& -i \omega m_{i} \widetilde{\Gamma}_{i}=n e[-\nabla \widetilde{\Phi}+i(\omega / c) \widetilde{A} \hat{b}]+(e / c) \widetilde{\Gamma}_{i} \times \mathbf{B}_{0} \\
& +\frac{c m_{e} v_{e i}}{4 \pi e} \nabla_{1}^{2} \widetilde{A} \hat{b}+\frac{3 m_{e} v_{e t}}{2 e} J_{\| 0}(r)\left(\frac{\widetilde{T}}{T}\right) \hat{b} \\
& -i \omega \tilde{n}_{e}+\nabla \cdot \widetilde{\Gamma}_{e}=0 \\
& -i \omega \tilde{n}_{i}+\nabla \cdot \Gamma_{i}=0
\end{aligned}
$$

where $\widetilde{\Gamma}_{e}$ and $\widetilde{\Gamma}_{i}$ are the perturbed electron and ion fluxes, $\hat{b} \equiv \mathbf{B} / B$, and the following relationship has been used:

$$
\widetilde{\mathbf{J}}_{\|}=(c / 4 \pi)(\nabla \times \nabla \times \widetilde{\mathbf{A}})_{\|}=-(c / 4 \pi) \nabla_{\perp}^{2} \tilde{A} \hat{b} .
$$

Two canceling terms, $\tilde{n} e\left(E_{0}-\eta_{0} J_{0}\right)$, have been omitted in the ion and electron momentum equations. The term $\sigma \nabla_{\|} \widetilde{T}$ is the thermal force of Braginski. This term arises from the temperature dependance of the collision frequency. In the presence of a gradient in the temperature the electrons coming from the hotter region of the plasma will feel less drag from the ions than those from the cooler region. The value of $\sigma$ is given by Braginski as 0.71 for $Z=1$, and it increases slowly with $Z$ until $\sigma=1.5$ for $Z=\infty$. For $Z=3-6, \sigma \approx 1$.

The equations are closed with the assumption of an equation of state,

$$
P \propto n^{\gamma},
$$

which leads to the following expression for $\widetilde{T} / T$ :

$$
\frac{\widetilde{T}}{T} \approx-(1-\gamma) \frac{\tilde{n}}{n}=-\frac{(1-\gamma)}{\gamma} \frac{\widetilde{P}}{P} .
$$

Based on experimental measurements, $\gamma \approx \frac{1}{2}$. A similar equation of state was used by Tang and Luhmann ${ }^{10}$ in a study of drift and drift-Alfvén waves in a linear arcjet device. They chose $\gamma=5 / 3$, which results in a temperature fluctuation in phase with the density fluctuations, in contrast to what was observed for the modes in Encore. Equations (8a)-(8d) and Eq. (10) form a closed set of equations for $\tilde{n}, \widetilde{\Phi}, \tilde{A}$ and the perpendicular electron and ion fluxes. These equations will now be reduced (with suitable approximations) to (i) the linearized parallel electron momentum equation, (ii) the ion response equation, and (iii) the quasineutrality condition, a set of three second-order differential equations in the variables $\tilde{n}, \widetilde{\Phi}$, and $\widetilde{A}$.

The parallel component of Eq. (8a) is

$$
\begin{aligned}
0= & -\left[-i k_{\|} \tilde{\Phi}+i(\omega / c) \tilde{A}\right]+i\left(\omega_{* p} / c\right) \tilde{A} \\
& -i k_{\|} \frac{\alpha \widetilde{P}}{\gamma n e}-\frac{c m_{e} v_{e i}}{4 \pi n e^{2}} \nabla_{\perp}^{2} \tilde{A}+\kappa_{0} \frac{\alpha \widetilde{P}}{\gamma n e},
\end{aligned}
$$


where the following definitions have been introduced (the prime indicates a derivative with respect to $r$ ):

$$
\begin{aligned}
& \omega_{*_{p}} \equiv-(m / r)\left[c\left(n T_{e}\right)^{\prime} / n e B\right], \\
& \kappa_{0} \equiv \frac{3(1-\gamma)}{2 \alpha} \frac{m_{e} v_{e i}}{n e} \frac{J_{i 0}(r)}{T}=\frac{3(1-\gamma)}{2 \alpha}\left(\frac{e E_{\|_{0}}}{T_{e}}\right), \\
& \alpha \equiv \gamma-\sigma(1-\gamma),
\end{aligned}
$$

and

$$
k_{\|}=\mathbf{k} \cdot \hat{b} \text {. }
$$

Here $\omega_{* p}$ is the electron diamagnetic drift frequency, $\kappa_{0}^{-1}$ is roughly the distance over which an electron picks up $T_{e}$ of energy from the loop voltage, and $k_{\|}$is the parallel component of the wavenumber. At a mode rational surface $k_{\|}$will go through zero. If $\kappa_{0} \approx k_{\|}$, then temperature fluctuations must be retained when calculating fluctuations in plasma current.

After rearranging, Eq. (11) becomes

$$
\begin{aligned}
& -i k_{\|} \tilde{\Phi}+i k_{\|} \frac{\alpha \widetilde{P}}{\gamma n e}-\kappa_{0} \frac{\alpha \tilde{P}}{\gamma n e} \\
& =\frac{i}{c}\left(\omega_{* p}-\omega+i \frac{m_{e} v_{e i} c^{2}}{4 \pi n e^{2}} \nabla_{1}^{2}\right) \tilde{A} .
\end{aligned}
$$

Defining a new variable,

$$
\widetilde{\Psi} \equiv \widetilde{\Phi}-\alpha \widetilde{P} / \gamma n e,
$$

so Eq. (13) becomes

$$
-\bar{k}_{\|} c \tilde{\Psi}=\left[\omega_{* p}-\omega+i\left(v_{e i} c^{2} / \omega_{p e}^{2}\right) \nabla_{\perp}^{2}\right] \tilde{A}-i \kappa_{0} c \tilde{\Phi},
$$

where

$$
\bar{k}_{\|} \equiv\left(k_{\|}+i \kappa_{0}\right) .
$$

Equation (15) describes how the induced electric field, the collisional drag, and the interaction of the equilibrium perpendicular current with fluctuating magnetic fields all act to modify the free movement of the electrons along the magnetic field. If the rhs of Eq. (15) were zero, $\widetilde{\Psi}$ would vanish, i.e., the electrons would free stream along the magnetic field, and would have a Boltzmann distribution, $\alpha \widetilde{P}$ / $\gamma \widetilde{P}=e \widetilde{\Phi} / T$. Thus Eq. (15) describes the extent to which the electrons do not have a Boltzmann distribution.

The ion response is found by solving Eq. (8b) for the divergence of the ion flux and then substituting this into the ion continuity equation, Eq. (8d). For argon plasmas the parallel ion flux terms, which give the ion sound speed corrections, are negligible and will not be included in this derivation. The linearized ion continuity equation, [Eq. (8d)], may then be written

$$
-\omega(\tilde{n} / n)=-(e / T)\left(\omega_{* n}+\omega \rho_{s}^{2} \nabla_{\perp}^{2}\right) \tilde{\Phi},
$$

where $\rho_{s}^{2}=T /\left(m_{i} \omega_{c i}^{2}\right)$. The first and second terms on the right correspond to the divergence of the $\mathbf{E} \times \mathbf{B}$ and polarization fluxes, respectively. Making use of Eqs. (10) and (14), this may be written

$$
-(\omega / \alpha) \widetilde{\Psi}=\left[\omega_{* n}-(\omega / \alpha)+\omega \rho_{s}^{2} \nabla_{1}^{2}\right] \widetilde{\Phi} .
$$

Quasineutrality implies that

$\nabla \cdot \widetilde{\mathbf{J}}=0$.
In terms of the perpendicular ion and electron fluxes and the parallel current, this becomes

$$
\nabla \cdot\left(\widetilde{\Gamma}_{1 i}-\widetilde{\Gamma}_{1 e}\right)=-(1 / e) \nabla \cdot \widetilde{\mathbf{J}}_{\|} \cdot
$$

The divergence of the perpendicular ion flux gives contributions from the $\mathbf{E} \times \mathbf{B}$ and polarization drifts. Likewise, the divergence of the perpendicular electron flux will give a contribution from the $\mathbf{E} \times \mathbf{B}$ drift, but will also have a contribution from the interaction of the equilibrium electron flow with the perturbed magnetic fields. (This last term drives the classical tearing instability. ${ }^{21}$ ) The divergence of the perpendicular electron flux is

$$
(i / n) \nabla \cdot \widetilde{\Gamma}_{1 e}=-(e / T)\left(\omega_{* n} \widetilde{\Phi}+\omega_{J} \widetilde{A}\right),
$$

where $\omega_{J}$ has been defined as

$$
\omega_{J} \equiv-4 \pi k_{\perp} \lambda_{d e}^{2}\left[J_{\| 0}^{\prime}(r) / B\right] \text {. }
$$

The contribution of the $\mathbf{E} \times \mathbf{B}$ drift to the perpendicular current is zero, and Eq. (19), with Ampère's law, Eq. (9), is then

$$
\begin{aligned}
(i / n) \nabla \cdot\left(\widetilde{\Gamma}_{1 i}-\widetilde{\Gamma}_{\perp e}\right) & =(e / T)\left(\omega_{J} \tilde{A}-\omega \rho_{s}^{2} \nabla_{1}^{2} \widetilde{\boldsymbol{\Phi}}\right) \\
& =-(i / n e) \nabla \cdot \widetilde{\mathbf{J}}_{\| i} \\
& =-(e / T) k_{\|} c \lambda{ }_{d}^{2} \nabla_{1}^{2} \widetilde{A} .
\end{aligned}
$$

Thus, the quasineutrality condition can be written as .

$$
\omega \rho_{s}^{2} \nabla_{\perp}^{2} \widetilde{\Phi}=k_{\|} c \lambda_{d}^{2} \nabla_{\perp}^{2} \widetilde{A}+\omega_{J} \widetilde{A} .
$$

\section{Simplification of the reduced equations}

Equations (15), (17), and (23) are a complete set of equations. They will now be rewritten in a form more convenient for the numerical and analytical work which follows. Using Eq. (15) to eliminate $\widetilde{\Psi}$ from Eq. (17) yields

$$
\begin{aligned}
& -\left(\omega / \alpha \bar{k}_{\|} c\right)\left\{\left[\omega_{* p}-\omega+i\left(v_{e i} c^{2} / \omega_{p e}^{2}\right) \nabla_{\perp}^{2}\right] \tilde{A}-i \kappa_{0} c \widetilde{\Phi}\right\} \\
& =\left(\omega / \alpha-\omega_{* n}-\omega \rho_{s}^{2} \nabla_{\perp}^{2}\right) \tilde{\Phi} .
\end{aligned}
$$

Equations (23) and (24) may be written in a more symmetric form. Using Eq. (23) to substitute for $\omega \rho_{s}^{2} \nabla_{1}^{2} \widetilde{\Phi}$ in Eq. (24) gives

$$
\begin{aligned}
& -\left(\omega / \alpha \bar{k}_{\| \mid} c\right)\left\{\left[\omega_{* p}-\omega+i\left(v_{e i} c^{2} / \omega_{p e}^{2}\right) \nabla_{\perp}^{2}\right] \tilde{A}-i \kappa_{0} c \widetilde{\Phi}\right\} \\
& =\left[(\omega / \alpha)-\omega_{* n}\right] \widetilde{\Phi}-\left(\omega_{J}+k_{\|} c \lambda_{d e}^{2} \nabla_{\perp}^{2}\right) \widetilde{A}
\end{aligned}
$$

This equation may now be solved for $\nabla_{1}^{2} \tilde{A}$ to get

$$
\begin{aligned}
\left(\alpha k_{\|} \bar{k}_{\|}-\right. & \left.i \frac{\omega v_{e i}}{V_{T e}^{2}}\right) \lambda_{d e}^{2} \nabla_{\perp}^{2} \widetilde{A} \\
& =\frac{\alpha \bar{k}_{\|}}{c}\left(\frac{\omega}{\alpha}-\omega_{* n}\right) \tilde{\Phi} \\
& +\left(\frac{\omega}{c^{2}}\left(\omega_{* p}-\omega\right)-\frac{\alpha \bar{k}_{\|}}{c} \omega_{J}\right) \tilde{A} \\
& -i \frac{\kappa_{0} c}{\omega}\left(\frac{\omega}{c}\right)^{2} \widetilde{\Phi},
\end{aligned}
$$

or, in a form similar to that of Ref. 4 ,

$$
\begin{aligned}
\nabla_{\perp}^{2} \widetilde{A}= & \sigma(r) \frac{\omega}{c}\left[\left(\frac{\omega}{c} \Omega_{2}-\alpha \frac{\omega_{J}}{\omega} \bar{k}_{\|}\right) \tilde{A}\right. \\
& \left.-\left(\alpha \Omega_{1} \bar{k}_{\|}+i \kappa_{0}\right) \widetilde{\Phi}\right],
\end{aligned}
$$

where the following definitions have been used: 


$$
\begin{array}{ll}
\Omega_{1}=\omega_{* n} / \omega-\frac{1}{\alpha}, & \Omega_{2}=\omega_{* p} / \omega-1, \\
\sigma(r)=\left[1 /\left(\alpha k_{\|} \bar{k}_{\|} D_{\|}-i \omega\right)\right]\left(4 \pi / \eta_{s}\right), & D_{\|}=v_{T}^{2} / \nu_{e i} .
\end{array}
$$

Here $D_{\|}$is the parallel electron diffusion coefficient, $\eta_{s}$ $\equiv m_{e} v_{e i} /\left(n e^{2}\right)$ is the Spitzer resistivity, and the function $\sigma(r)$ is the generalized dimensionless conductivity in the semicollisional ordering of Drake and Lee, ${ }^{22}$ sometimes referred to as the semihydrodynamic approximation. ${ }^{6}$

A similar equation can be written for $\nabla_{1}^{2} \widetilde{\Phi}$. Equation (27) is used to substitute for $\nabla_{1}^{2} \tilde{A}$ in Eq. (23), resulting in

$$
\begin{gathered}
\lambda_{d e}^{2} \sigma(r) \frac{\omega}{c}\left[\left(\frac{\omega}{c} \Omega_{2}-\frac{\alpha \omega_{J}}{\omega} \bar{k}_{\|}\right) \widetilde{A}-\left(\alpha \Omega_{1} \bar{k}_{\|}+i \kappa_{0}\right) \widetilde{\Phi}\right] \\
=\left(1 / k_{\|} c\right)\left(\omega \rho_{s}^{2} \nabla_{\downarrow}^{2} \widetilde{\Phi}-\omega_{J} \widetilde{A}\right),
\end{gathered}
$$

which, with the previous definitions, may be written as

$$
\begin{aligned}
\nabla_{\perp}^{2} \widetilde{\Phi}= & -\sigma(r)\left(\frac{V_{\mathrm{A}}}{c}\right)^{2}\left[\left(\alpha \Omega_{1} k_{\|} \bar{k}_{\|}+i \kappa_{0} k_{\|}\right) \widetilde{\Phi}\right. \\
& \left.-\left(\frac{\omega}{c} \Omega_{2} k_{\|}-i \frac{\omega_{J}}{D_{\|}}\right) \tilde{A}\right] .
\end{aligned}
$$

The final step is to use Eqs. (15) and (27) to find a simple equation for the density fluctuations in terms of $\widetilde{\Phi}$ and $\widetilde{A}$. Substituting for $\nabla_{1}^{2} \widetilde{A}$ in Eq. (15)

$$
\begin{aligned}
-\frac{\bar{k}_{\|} c}{\omega} \widetilde{\Psi}= & \Omega_{2} \widetilde{A}-\frac{i \kappa_{0} c}{\omega} \widetilde{\Phi} \\
& +i\left(\frac{\eta_{s} c^{2}}{4 \pi \omega}\right) \sigma(r) \frac{\omega}{c}\left[\left(\frac{\omega}{c} \Omega_{2}-\alpha \frac{\omega J}{\omega} \bar{k}_{\|\|}\right) \tilde{A}\right. \\
& \left.-\left(\alpha \Omega_{1} \bar{k}_{i \|}+i \kappa_{0}\right) \widetilde{\Phi}\right]
\end{aligned}
$$

which may then be simplified further:

$$
\begin{aligned}
-\widetilde{\Psi}= & \alpha \lambda_{d \mathrm{e}}^{2} \sigma(r)\left[\left(\frac{\omega}{c} \Omega_{2} k_{\|}-i \frac{\omega_{J}}{D_{\|}}\right) \tilde{A}\right. \\
& \left.-\left(i \kappa_{0} k_{\|}+i \frac{\omega}{D_{\|}} \Omega_{1}\right) \tilde{\Phi}\right] .
\end{aligned}
$$

Equations (27), (30), and (32) make up the final set of equations which will be used in the numerical code as discussed in the following sections.

\section{Approximate analytical solution}

Before proceeding with the discussion of the numerical results, an approximate analytical solution to Eqs. (27), (30), and (32) will be found. This will provide a check on the numerical work which follows. An analytic dispersion relation may be found under the following assumptions: (i) the density profile is Gaussian, (ii) the electron temperature is constant, (iii) the plasma current profile is flat, i.e., $k_{\|}=$const and (iv) the dependance of the Alfvén velocity on minor radius can be neglected. With these approximations, $\omega_{* n}=\omega_{* p}$ $=$ const., $\alpha=1, \Omega_{1}=\Omega_{2}=\Omega, \omega_{J}=0$, and $\bar{k}_{\|}=k_{\|}$and so Eqs. (27) and (30) become

$$
\begin{aligned}
& \rho_{s}^{2} \nabla_{\perp}^{2} \tilde{\Phi}=-k_{\|}^{2} \lambda_{d e}^{2} \sigma(r) \Omega\left[\tilde{\Phi}-\left(\omega / k_{\|} c\right) \tilde{A}\right], \\
& \rho_{s}^{2} \nabla_{\perp}^{2} \tilde{A}=-\frac{c^{2}}{V_{A}^{2}} k_{\|} \lambda_{d e}^{2} \sigma(r) \Omega\left(\frac{\omega}{c}\right)\left[\tilde{\Phi}-\left(\frac{\omega}{k_{\|} c}\right) \tilde{A}\right],
\end{aligned}
$$

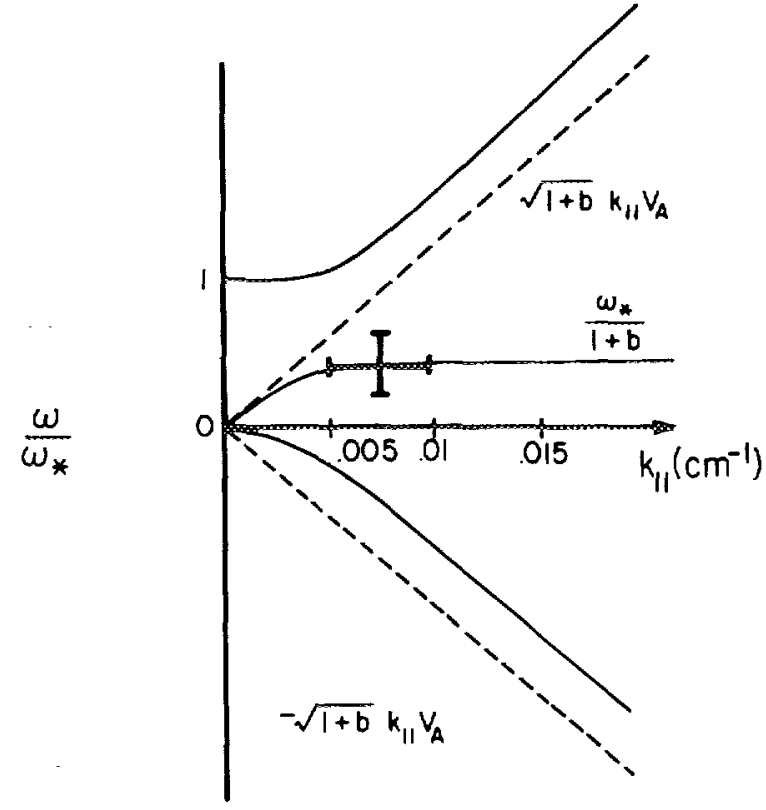

FIG. 10. The analytic dispersion relation showing the coupling of the drift and Alfvén branches at small $k_{i k}$. The error bars show the location of the observed frequency and $k_{\mathrm{fl}}$. The width of the error bars reflects the variation of $k_{\sharp}$ and $\omega$. over the range of minor radius where the mode has an appreciable amplitude (cf. Figs. 7 and 11). This figure is plotted from Eq. (40), assuming $b=1.5$. The observed frequency of the mode is $5 \mathrm{kHz}$ and the electron diamagnetic drift frequency is between 10 and $20 \mathrm{kHz}$.

where all coefficients are now constant. These equations may also, for simplicity, be written in a matrix form as

$\rho_{s}^{2} \nabla_{\perp}^{2}\left[\begin{array}{c}\tilde{\Phi} \\ \tilde{A}\end{array}\right]+\Omega f\left[\begin{array}{cc}1 & -\frac{\omega}{k_{\|} c} \\ \frac{\omega c}{k_{\|} V_{\mathrm{A}}^{2}} & -\left(\frac{\omega}{k_{\|} V_{\mathrm{A}}}\right)^{2}\end{array}\right]\left[\begin{array}{l}\tilde{\Phi} \\ \tilde{A}\end{array}\right]=0$,

where

$$
f \equiv k_{\|}^{2} \lambda_{d e}^{2} \sigma(r)=1 /\left(1-i \omega \tau_{\|}\right), \quad \tau_{\|} \equiv 1 / k_{\|}^{2} D_{\|} .
$$

The eigenvalues for the matrix are

$$
\lambda_{1,2}=\left\{\begin{array}{c}
{\left[1-\left(\omega / k_{\|} V_{\mathrm{A}}\right)^{2}\right]} \\
0
\end{array}\right.
$$

so if the appropriate transformations to diagonalize the matrix are carried out, the result is two decoupled equations of the form

$$
\begin{aligned}
& \rho_{s}^{2} \nabla_{\perp}^{2}\left(\tilde{\Phi}-\frac{\omega}{k_{\|} c} \tilde{A}\right) \\
& \quad+\Omega f\left[1-\left(\frac{\omega}{k_{\|} V_{\mathrm{A}}}\right)^{2}\right]\left(\tilde{\Phi}-\frac{\omega}{k_{\|} c} \tilde{A}\right)=0, \\
& (\Omega f)^{-1} \rho_{s}^{2} \nabla_{\perp}^{2}\left(-\frac{\omega c}{k_{\|} V_{\mathrm{A}}^{2}} \tilde{\Phi}+\tilde{A}\right)=0 .
\end{aligned}
$$

Equation (38) has solutions of the form $e^{i m \theta} J_{m}\left(k_{r} r\right)$, and the standard ${ }^{23}$ drift-Alfvén dispersion relation is then recovered:

$$
\left(1-i \omega \tau_{\|}\right) \omega k_{r}^{2} \rho_{s}^{2}=\left(\omega_{*}-\omega\right)\left[1-\left(\omega / k_{\|} V_{\mathrm{A}}\right)^{2}\right],
$$

where $k_{r}$ is defined as $x_{j}^{m} / a$ and $x_{j}^{m}$ is the $j$ th zero of the $m$ th Bessel function. Solutions to Eq. (40) are graphed in Fig. 10, 
with the assumption $\omega \tau_{\|}<1$ and that $b=1.5$. Equation (40) is cubic in $\omega$; the three roots, with the assumptions that $\omega \tau_{\mathrm{H}}$ $<1$ and $k_{\| \mid} V_{\mathrm{A}}>\omega_{*}$, are given as

$$
\begin{aligned}
& \omega_{1} \approx \frac{\omega_{*}}{1+b}\left(1-\frac{\omega_{*}^{2}}{\omega_{\mathrm{A}}^{2}} \frac{b}{(1+b)^{3}}\right)+i \frac{\omega_{*}^{2} \tau_{\|} b}{(1+b)^{3}}, \\
& \omega_{2,3} \approx \pm(1+b)^{1 / 2} \omega_{\mathrm{A}}+[b / 2(1+b)] \omega_{*}-\frac{1}{2} i b \omega_{\mathrm{A}}^{2} \tau_{\|},
\end{aligned}
$$

where $\omega_{\mathrm{A}} \equiv k_{\|} V_{\mathrm{A}}$ and $b \equiv k_{r}^{2} \rho_{s}^{2}$. These solutions correspond to the two shear-Alfvén solutions and the electrostatic drift wave solution. Equation (39) has just one root, namely, $\omega=0$ (i.e., $\Omega \rightarrow \infty$ ). [If the current gradient terms are kept, this solution has a nonzero frequency $\left(\omega \approx 10^{2} \mathrm{~Hz}\right)$, but still too slow to be seen on the time scale of the plasma existence.]

If the modes described by Eq. (40) exist [i.e., $\omega$ is given by Eqs. (41) or (42)], then the only way to satisfy Eq. (39) is to set

$$
\widetilde{A}=\left(\omega c / k_{\|} V_{\mathrm{A}}^{2}\right) \widetilde{\Phi}
$$

Another relation between $\tilde{A}$ and $\widetilde{\Phi}$ can be deduced as follows: Eq. (33) reduces to the electrostatic wave dispersion relation when $\widetilde{A}=0$, thus the coupling of the drift to the Alfven branch is seen to be important when the inductive component of the parallel electric field becomes comparable to the electrostatic component, i.e.,

$$
(\omega / c) \widetilde{A} \approx k_{\|} \tilde{\Phi} \text {. }
$$

From Eqs. (43) and (44), the condition for significant coupling of the drift to the Alfvén waves is that the parallel phase velocity of the drift wave approaches the Alfven velocity, i.e.,

$$
\left\{\omega / k_{\|} V_{\mathrm{A}}\right)^{2} \approx 1,
$$

or, in the terms of the plasma beta,

$$
\beta_{T} \approx\left(k_{\|} V_{T_{e}} / \omega\right)^{2}\left(m_{e} / m_{i}\right)
$$

It is the normalized magnetic fluctuation level $\widetilde{B} / B$ that is generally of interest, so using $\widetilde{B}_{r} \approx i k_{1} \widetilde{A}$ and $\beta_{T}=8 \pi n T_{e} /$ $B^{2}$, Eq. (43) becomes

$$
\frac{\widetilde{B}}{B} \approx \frac{i}{2} \frac{k_{1}}{k_{\|}} \frac{\omega}{\omega_{\mathrm{ci}}} \beta_{T}\left(\frac{e \widetilde{\Phi}}{T}\right),
$$

thus regaining the result derived by Kadomtsev. ${ }^{23}$

Fluctuations in the electron temperature, not included in the above equations, can also be important in the coupling

TABLE I. This table compares the experimental peak amplitudes, phases, and frequencies of the radial eigenfunctions for $e \widetilde{\Phi} / T$ and $e \widetilde{A} / T$ to those

\begin{tabular}{|c|c|c|c|c|}
\hline & $\begin{array}{c}\text { nparison of theo } \\
n \tilde{n} / n \\
\text { (amp. phase) }\end{array}$ & $\begin{array}{l}\text { oretical predict } \\
\qquad e \bar{\Phi} / T \\
\text { (amp. phase) }\end{array}$ & $\begin{array}{c}\text { tions to experim } \\
\qquad \vec{A} / T \\
\text { (amp. phase) }\end{array}$ & $\begin{array}{l}\omega_{r} \\
\left(10^{4} \mathrm{rad} / \mathrm{sec}\right)\end{array}$ \\
\hline $\begin{array}{l}\text { experiment } \\
\text { drift wave }\end{array}$ & $\begin{array}{l}\left(0.40,0^{\circ}\right) \\
\left(0.40,0^{\circ}\right)\end{array}$ & $\begin{array}{l}\left(0.20,45^{\circ}\right) \\
\left(0.16,10^{\circ}\right)\end{array}$ & $\begin{array}{c}\left(30,-100^{\circ}\right) \\
\left(28,20^{\circ}\right)\end{array}$ & $\begin{array}{l}3.0 \\
2.4\end{array}$ \\
\hline Alfvén & {$\left[\begin{array}{l}(0.40, \cdots) \\
(0.40, \cdots)\end{array}\right.$} & $\begin{array}{l}(0.40, \cdots) \\
(0.40, \cdots)\end{array}$ & $\begin{array}{l}(800, \cdots) \\
(70, \cdots)\end{array}$ & $\begin{array}{r}30.0 \\
-15.0\end{array}$ \\
\hline
\end{tabular}
predicted theoretically for the drift wave and two shear-Alfvén waves. The numerical code is based on a linear theory, so the peak amplitude of $\bar{n} / n$ is used to scale the theoretical results. Likewise, the relative phases are measured with respect to $\tilde{n} / n$. The phases of the eigenfunctions for the two Alfvén solutions vary considerably over the minor radius: thus the relative phases of $\tilde{n} / n, e \widetilde{\Phi} / T$, and $e \tilde{A} / T$ are not well defined. of the drift to the Alfvén branch. The electron temperature fluctuations affect the local plasma conductivity, and can thus induce fluctuations in the local current density. The condition that this effect be important is that the effective force on the electrons from the change in conductivity be comparable to the force from the electric field of the drift wave. The exact condition that temperature fluctuations be important is

$$
\kappa_{0} \equiv[3(1-\gamma) / 2 \alpha]\left(e E_{\| 0} / T\right) \geqslant k_{\|},
$$

which means that the loop voltage $V$, must be of the order of the electron temperature. As $V_{l} \approx T$ in Encore, temperature fluctuations have been included in the model, as discussed earlier. This effect will not be important in hotter tokamaks where $V_{l}<T$.

\section{E. Numerical solution of the equations}

The full set of equations is solved for various plasma parameters using a numerical code. The code uses as input the standard plasma parameters such as toroidal field, $\boldsymbol{Z}_{\mathrm{eff}}$, ion mass, etc. It also uses multi-parameter analytic functions to approximate the experimentally determined density and current profiles. The code uses the conductivity temperature profile, rather than the somewhat different profile obtained from the Langmuir probe measurements. The equations are not strongly sensitive to the temperature profiles and the conductivity profile is more convenient to use.

The problem is a boundary value problem, and the eigenvalues and functions are affected, to some extent, by the boundary conditions used. As the vacuum chamber wall is a very good conductor, conducting wall boundary conditions are used, i.e., (without temperature fluctuations) $\widetilde{\Phi}(a)=\widetilde{A}(a)=0$. Experimentally it is observed that $\widetilde{B}_{r}$, and hence $\widetilde{A}$, does go to zero at the wall, but that $\tilde{n} / n$ does not. The sensitivity of the solutions to the boundary conditions was checked by using several boundary conditions at the wall on $\widetilde{\Phi}$, but requiring always that $\widetilde{A}$ go to zero. It was found that the effects on the frequency and mode amplitudes were small, typically less than a few percent.

\section{F. Results}

Numerical solutions have been found corresponding to the drift wave and the two shear-Alfvén solutions of the analytic dispersion relation, Eq. (40). The results of these numerical calculations for parameters consistent with the data presented in Figs. 4-9 are presented in Table I and Fig. 11. As the code is based on a linear theory and does not predict a saturation level, the numerical results must be scaled to one of the experimentally measured quantities; in this case, as $\tilde{n}$ / $n$ is the best known of the experimental measurements, it was chosen as the point of comparison. From the data in Table I, it is obvious that the experimental measurements are best fit by the drift-wave solution. The two shear-Alfvén solutions have much larger ratios of vector potential to density fluctuations and the frequencies for the shear-Alfvén solutions do not agree with the observed frequency of the mode.

The profiles of the safety factor, density, and electron temperature used for this solution are presented in Fig. 4. The predicted frequency of the mode was found to depend 

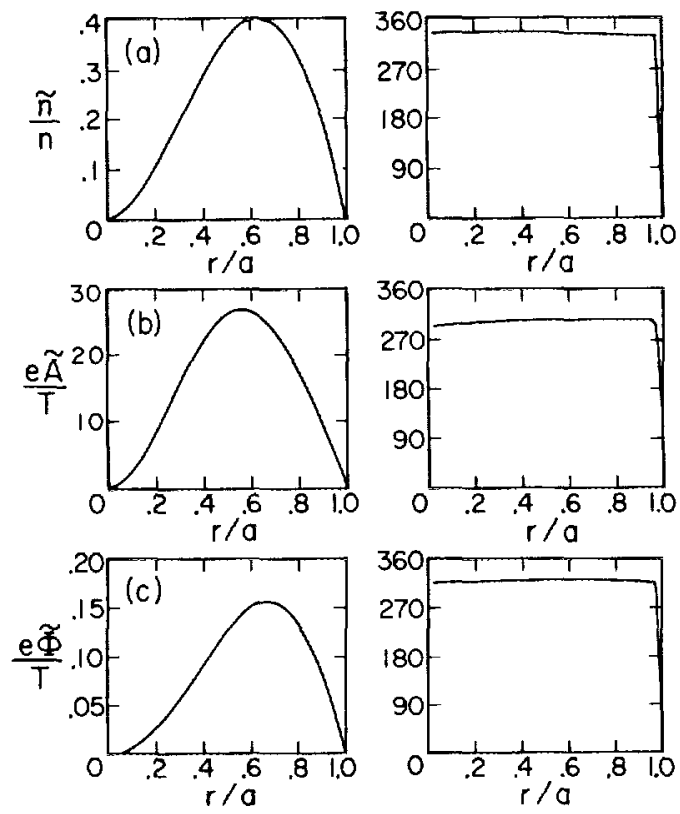

FIG. 11. Radial profiles of the theoretically computed amplitude and phase of the (a) density, (b) vector potential, and (c) space potential fluctuations for the $m=2$ drift -wave solution. The amplitudes have been set by normalizing the density fluctuations to $\tilde{n} / n=0.4$. The space and vector potential fluctutions are normalized to the local average electron temperature.

strongly on the choice of $Z_{\text {eff }}\left(\omega_{*} \propto T \propto Z^{2 / 3}\right)$. The $Z_{\text {eff }}$ was chosen based on a comparison of the conductivity temperature [see Eq. (2)] and the temperature determined from the Langmuir probe $I-V$. The data indicates, as mentioned earlier, that $Z_{\text {eff }}$ is a function of minor radius, and thus there is some uncertainty introduced in the choice of a constant $Z_{\text {eff }}$ in the code. For this data the choice of $Z_{\text {eff }}=4.5$ was made to give a reasonable "average" fit of the conductivity and Langmuir probe temperatures.

The other parameter that was determined from the experimental data was the ratio of $\widetilde{T} / T$ to $\tilde{n} / n$. The measurements of the saturated mode gave this ratio as $\widetilde{T} /$ $T=-0.3 \tilde{n} / n$. The temperature fluctuations were found to have a stabilizing effect on the mode. If the temperature fluctuations were set equal to zero, the mode was unstable with a growth rate of $\gamma=1.3 \times 10^{3} / \mathrm{sec}$. Inclusion of the temperature fluctuations resulted in a negative growth rate. If the temperature fluctuations are caused by local radiative cooling, they would scale nonlinearly with $\tilde{n} / n$ and would be a possible saturation mechanism for the mode.

With the above choices of parameters, the numerical results agree quite well with the experimental measurements as can be seen in Table $I$. The predicted frequency agrees quite well with the measured value, experimentally $f=5.0$ $\mathrm{kHz}$ and theoretically $f=4.0 \mathrm{kHz}$. As discussed above, the uncertainties in the value of $Z_{\text {eff }}$ mean that the predicted frequency is only good to $\approx 1 \mathrm{kHz}$. The predictions of the relative amplitudes of $\tilde{n} / n, e \widetilde{\Phi} / T$, and $\widetilde{A}$, which were not as sensitive to the choice of $Z_{\text {eff }}$, were quite good also.

The predictions of the relative phases between the fluctuating quantities were not good. The theoretical phase shifts between $\tilde{n} / n, e \widetilde{\Phi} / T$, and $\widetilde{A}$ were all quite small. Experimentally, it was found that the space potential fluctuations led the density fluctuations by $\approx 45^{\circ}$ and that the vector potential fluctuations lagged the density fluctuations by $\approx 100^{\circ}$. The phase shifts (at least between $\tilde{n} / n$ and $e \bar{\Phi} / T$ ) are related to the stability of the mode and the theory has neglected at least one important destabilizing effect, the resonant interaction of the waves with the current-carrying electrons. ${ }^{24}$ It seems unlikely, however, that inclusion of this would resolve the above discrepency in the phases.

\section{SUMMARY AND DISCUSSION}

The saturated state of a low-frequency, coherent global mode has been studied in the Encore tokamak using probe techniques. The mode is found to have large fluctuations in density, electron temperature, space potential, and magnetic field. The poloidal and toroidal mode number $(m=2$, $n=1$ ), as well as the radial structure of the fluctuations have been measured. The temperature fluctuations are found to be out of phase with the density fluctuations, consistent with expectations for a radiation dominated discharge. The space potential fluctuations are found to lead the density fluctuations by $\approx 45^{\circ}$, indicating that the mode is transporting plasma radially inward.

The equilibrium plasma characteristics were also determined with probe measurements. Magnetic probes were used to determine the radial profile of the poloidal magnetic field, from which the rotational transform and current density profiles could be deduced. A Langmuir probe was used to measure radial profiles of the density, electron temperature, and space potential. The space potential was nearly constant in the central region of the plasma, indicating that there was almost no $\mathbf{E} \times \mathbf{B}$ rotation of the plasma, i.e., the observed frequency of the mode was not Doppler shifted.

The experimental measurements were compared with the predictions of a code developed for the purpose. The code is based on a linear, two-fluid theory of the coupling of drift and shear-Alfvén modes. Of the two shear-Alfvén solutions and the drift branch solution, it was found that the drift wave solution best fit the observed frequency of the mode and the relative amplitudes of the density, space potential, and magnetic fluctuations. The shear-Alfvén solutions had much larger magnetic fluctuations for a given level of density fluctuations. The identification of the mode as a finite $\beta_{T}$ modified drift wave means that the mode is more closely related to the higher frequency, turbulent fluctuations observed on larger machines, rather than the lower frequency coherent Mirnov oscillations.

The Mirnov oscillations observed on larger tokamaks have many features in common with the finite beta modified drift wave observed in Encore, but the Mirnov oscillations are not likely to be drift waves. In larger tokamaks with hot, lighter ions, the ion thermal velocity becomes comparable to the parallel phase velocity of the mode a short distance from the rational surface. This would lead to ion Landau damping of the mode. Further, estimates of the ratio of magnetic to density fluctuations of larger machines, using Eq. (47), show that the magnetic fluctuation level would be too small by several orders of magnitude to explain the Mirnov oscillations. Using $T_{e}=1 \mathrm{keV}, B_{T}=30 \mathrm{kG}, n=2 \times 10^{13} \mathrm{~cm}^{-3}$, and $r=40 \mathrm{~cm}$, then $\omega_{*} \sim 4 \times 10^{3}, \omega_{\mathrm{ci}} \sim 3 \times 10^{8}, \beta_{T} \sim 0.002$, 
and $k_{\perp} /\left(2 k_{\|}\right) \sim 10$, or

$$
\widetilde{B} / B \approx 3 \times 10^{-7}(e \widetilde{\Phi} / T) \text {. }
$$

A similar calculation for the Encore tokamak would give

$$
\widetilde{B} / B \approx 10^{-2}(e \widetilde{\Phi} / T) \text {. }
$$

Thus the large magnetic fluctuations associated with this drift wave are caused in part by the relatively large ratio of $\omega / \omega_{c i}$ and to a moderate plasma $\beta_{T} \approx .01$.

\section{ACKNOWLEDGMENTS}

This work was taken from the doctoral dissertation of one of the authors (E. D. F). One of the authors (E. D. F.) would like to thank Paulett Liewer and Stuart Zweben for many helpful discussions during the course of this work.

This work was supported in part by U. S. Department of Energy Contract No. DE-FG03-84ER53173.

'P. C. Liewer, submitted to Nucl. Fusion.

${ }^{2}$ D. A. Monticello and R. B. White, Phys. Fluids 23, 366 (1980).

${ }^{3}$ J. F. Drake and R. Kleva, Phys. Fluids 24, 1499 (1981).

${ }^{4}$ N. T. Gladd, J. F. Drake, C. L. Chang, and C. S. Liu, Phys. Fluids 23, 1182 (1982).

${ }^{5}$ K. T. Tsang, J. C. Whitson, and J. Smith, Phys. Fluids 22, 1689 (1979).
'S. M. Majahan, R. D. Hazeltine, H. R. Strauss, and D. W. Ross, Phys. Fluids 22, 2147 (1979).

${ }^{2} \mathrm{G}$. Schmidt, Physics of High Temperature Plasmas (Academic, New York, 1979), 2nd ed., p. 354

${ }^{8}$ T. J. M. Boyd and J. J. Sanderson, Plasma Dynamics (Barnes and Noble, New York, 1969), p. 83

${ }^{9}$ Y. Nishida and K. Ishii, Phys. Rev. Lett. 33, 352 (1974)

${ }^{10} J$. T. Tang and N. C. Luhmann, Jr., Phys. Fluids 19, 1935 (1976).

"D. E. Post, R. V. Jensen, C. B. Tarter, W. H. Grasberger, and W. A. Lokke (private communication)

${ }^{12} \mathrm{G}$. Bateman, MHD Instabilities (MIT, Cambridge, 1978), p. 129.

${ }^{13}$ A. B. Hassam, Phys. Fluids 23, 38 (1980).

${ }^{14}$ L. Spitzer, Jr., and R. Harm, Phys. Rev. 89, 977 (1953).

${ }^{15}$ J. R. Sanmartin, Phys. Fluids 13, 103 (1970).

${ }^{16} \mathrm{~V}$. D. Shavranov, in Reviews of Plasma Physics, edited by M. A. Leontovich (Consultants Bureau, New York, 1966), Vol. 2, p. 103.

${ }^{17}$ B. B. Kadomtsev and O. P. Pogutse, in Reviews of Plasma Physics, edited by M. A. Leontovich (Consultants Bureau, New York, 1966), Vol. 5, p. 284.

${ }^{18}$ H. W. Hendel, T. K. Chu, and P. A. Politzer, Phys. Fluids 11, 2426(1968).

${ }^{19}$ R. F. Ellis and R. W. Motley, Phys. Fluids 17, 582 (1974).

${ }^{20}$ S. I. Braginski, in Reviews of Plasma Physics, edited by M. A. Leontovich [Consultants Bureau, New York, 1965), Vol. 1, p. 214.

${ }^{21}$ H. P. Furth, P. H. Rutherford, and H. Selberg, Phys. Fluids 16, 1054 [1973].

22J. F. Drake and Y. C. Lee, Phys. Fluids 20, 1341 (1977).

${ }^{23}$ B. B. Kadomtsev, Plasma Turbulence (Academic, New York, 1965), pp. 82-83.

${ }^{24}$ N. T. Gladd and C. S. Liu, Phys. Fluids 22, 1289 (1979). 